\title{
La notion de compétences relationnelles : une conception utilitariste de la relation à l'autre
}

\section{Odile Camus}

\section{(2) OpenEdition}

\section{Journals}

Édition électronique

URL : http://journals.openedition.org/communicationorganisation/3585

DOI : 10.4000/communicationorganisation.3585

ISSN : $1775-3546$

\section{Éditeur}

Presses universitaires de Bordeaux

\section{Édition imprimée}

Date de publication : 1 décembre 2011

Pagination : 127-140

ISBN : 978-2-86781-745-8

ISSN : $1168-5549$

\section{Référence électronique}

Odile Camus, «La notion de compétences relationnelles : une conception utilitariste de la relation à

l'autre », Communication et organisation [En ligne], 40 | 2011, mis en ligne le 01 décembre 2014,

consulté le 10 décembre 2020. URL : http://journals.openedition.org/communicationorganisation/ 3585 ; DOI : https://doi.org/10.4000/communicationorganisation.3585 


\title{
La notion de compétences relationnelles : une conception utilitariste de la relation à l'autre
}

\author{
Odile Comus ${ }^{1}$
}

\section{La notion de " compétences relationnelles et sociales" (CRS) 2 : un peu plus de confusion dans le domaine problématique de l'évaluation des compétences?}

Quel article traitant de compétences ne commence-t-il pas par souligner la polysémie du terme et le flou conceptuel entourant la notion ? Ajoutant à la confusion, les CRS sont devenues incontournables, - souvent considérées comme clef de l'employabilité, et base de la réussite professionnelle. Leur désignation est de plus multiple : sociales ou relationnelles (ce deuxième terme étant d'un usage plus récent), mais aussi compétences comportementales, ou professionnelles (entendu comme transversales), ou encore savoir faire relationnel (substitut direct du savoir être), et même "volonté de travailler " ou « volontés professionnelles ». Par delà la diversité croissante des pratiques d'évaluation professionnelle et de leurs finalités, et partant le foisonnement des définitions et des références impliquant de multiples disciplines, est-il possible de trouver quelques lignes transversales pour appréhender l'éventuelle consistance de la notion?

Le modèle des compétences possède certes une consistance interne forte et maintes fois décrite, qui lui est conférée par sa fonction idéologique notamment mise en évidence dans l'analyse de ses conditions d'émergence (sous-emploi, dévalorisation des acquis scolaires, etc...). Cette notion serait nécessaire à la reproduction d'un modèle entrepreneurial qui ne chercherait pas tant l'objectivité dans l'évaluation des personnes, que, finalement, le maintien et la légitimation d'une hiérarchie professionnelle reflétant la

1. Odile Camus est Maître de Conférences HDR en psychologie sociale à l'Université de Rouen. Ses travaux portent sur les processus idéologiques appréhendés du point de vue d'une psychologie sociale de la communication. Cette étude intervient dans le cadre du contrat ANR 08-COMM-043. "Savoir communiquer »: approche critique de l'efficacité persuasive ; odile.camus@remuements.net.

2. L'usage actuel tendant à les confondre, nous avons choisi de ne pas les distinguer ici. Cela étant la notion de compétence(s) sociale(s) reste plus fréquente dans une acception large incluant tous les contextes de la vie, tandis que celle de compétences relationnelles (toujours au pluriel d'ailleurs) reste plus spécifique au contexte professionnel. 
hiérarchie sociale. De ce point de vue, la notion même de compétence prend ancrage dans le modèle normatif de l'individualisme libéral tel que décrit en particulier dans Beauvois (2005). Ce modèle en effet favorise l'occultation des déterminismes sociaux au profit d'une mise en cause des personnes dans leurs parcours professionnel (en particulier : domaine de l'insertion).

Il n'en reste pas moins que le champ des CRS ressemble à une mosaïque de caractéristiques disparates. Sera examiné ici l'usage de la notion dans des contextes variés, constitués en quelque sorte en "formation discursive " (Foucault 1969). La notion de formation discursive permet de rendre compte de l'organisation sous-jacente (« ensemble de règles ») à un ensemble d'énoncés a priori hétéroclites, voire contradictoires, sur un objet donné. Cette notion présuppose une cohérence entre tous les discours co-occurrents dans une " épistémé » donnée - quel que soit leur statut au regard des savoirs légitimes. Les textes scientifiques retiendront donc notre attention au même titre que les productions les plus visiblement marquées idéologiquement. À notre sens, et en dépit des distances de Foucault d'avec la notion d'idéologie, cette cohérence rend compte de l'idéologie au sens strict du terme, et une approche en termes de formation discursive permet d'en saisir la face obscure, celle des évidences non problématisées.

\section{La signification de la notion de CRS : de la polysémie manifeste à la cohérence implicite}

\section{Les définitions dans les offres de formation et d'évaluation}

Le premier corpus est constitué de 21 offres de formation et d'évaluation en matière de CRS, offres émanant des prestataires les plus variés (organismes de bilan de compétences, conseils en communication, coaching, etc. Voir annexe). L'analyse, qualitative et articulant analyse de contenu et analyse du discours, est focalisée sur les référents des CRS et leurs interrelations, ainsi que sur leur expression lexicale. Le format prototypique de ces offres peut être succinctement décrit de la manière suivante : énumération de caractéristiques variées en guise et place de définition; usage systématique, bien que non exclusif, du lexique personnologique ; absence de références théoriques (psychologiques notamment)... On ne peut y trouver matière pour une définition scientifique des CRS. L'analyse de ce corpus révèle une première stratégie de construction de la cohérence, reposant sur l'intrication entre énoncés descriptifs et évaluatifs : le registre évaluatif, normatif et essentialiste, de la personnologie (voir Beauvois 1984, 1994) d'une part, et le registre descriptif des comportements avec autrui, d'autre part, s'y trouvent entremêlés, voire confondus. La valeur normative des CRS est ainsi omniprésente dans les références aux conduites relationnelles attendues, lesquelles se présentent formellement comme des catégories descriptives opérationnelles, mais sans qu'il soit effectivement possible d'attribuer sans équivoque, sur la base de ces 
pseudo-descriptions, une CRS donnée à partir d'un comportement donné. Le document 1 illustre cette stratégie.

\section{Document 1}

\section{Valeur normative et confusion description/évaluation dans le corpus des offres (extraits des définitions proposées)}

Sont soulignés par nous dans ces exemples les termes évaluatifs pour lesquels la mise en relation avec des faits observables paraît la plus problématique.

- Relation aux autres :

Développer l'aptitude à aborder les autres de manière ouverte, à entrer en relation avec eux de manière flexible. (Monika Radecki)

Nous définirons cette compétence comme la capacité d'entrer en contact, d'adopter et d'adapter, vis-à-vis d'autrui et en fonction des buts que nous nous sommes fixés, des comportements, aptitudes et attitudes efficaces (...) afin d'entretenir des échanges satisfaisants et fructueux dans un contexte professionnel. (Solutions \& Co)

Eprouver un intérêt réel pour les soucis des autres. (Accompagnement Conseil RH)

- Persuasion :

Envoyer des messages clairs et convaincants. (Accompagnement Conseil RH) S'exprimer de façon convaincante et vivante. (LCA)

Acquérir les bases d'une communication saine et efficace augmente la productivité. (TQ16)

\section{- Image de soi :}

Le déploiement de la personnalité comme nécessité à l'action autonome. (Michel Pretre)

Communiquer le plus clairement possible et avec conviction (...) ses projets personnels de développement en donnant une image positive de soi. (PMC) S'affirmer dans ses relations transversales en exprimant ses critiques de façon constructive. (Cegos)

Maintenir une attitude et une posture positive face au travail et à la vie. (PMC)

- Rapport à l'entreprise :

La faculté de vivre et de travailler dans la société en prenant des responsabilités et en participant activement à son développement. (Michel Pretre)

Une seconde stratégie concerne la structure globale de ces offres, sous-tendue par des associations conceptuelles implicites. Plus précisément, par delà la diversité des descriptions définitoires, l'ensemble des CRS citées peut faire l'objet d'un regroupement en quelques dimensions consensuelles. Ces dimensions y sont fréquemment distinguées de façon explicite ; elles rassemblent différentes caractéristiques d'apparence homogène ; elles sont sémantiquement définies de façon proche d'une offre à l'autre. Mais leur 
homogénéité repose en fait sur des associations arbitraires entre caractéristiques. De plus, la distinction entre dimensions est principalement formelle, des référents proches pouvant se retrouver dans plusieurs dimensions :

- Affects orientés vers autrui (empathie, perception du sentiment des autres, respect, tolérance...). En même temps, sont incisés dans cette référence des éléments relatifs au potentiel d'influence sur autrui, introduisant implicitement une visée utilitariste qui n'en paraît pas dissociable.

- Rapport à soi (confiance en soi, auto-évaluation, conscience de soi, maturité d'esprit, développement personnel...). Cette référence recouvre en partie la précédente, de par des incises de même nature (visée utilitariste implicite).

- Travail en équipe (coopération, collaboration, négociation, résolution de conflits, synergie de groupe, objectifs communs...). La référence à l'influence sur autrui est ici plus explicite que dans les autres dimensions, et se décline selon deux axes : l'impression produite, et le potentiel de leadership.

\section{Document 2}

\section{L'influence sur autrui, noyau structurant les CRS}

\section{L'empathie utilitariste :}

- Savoir déchiffrer les flux émotionnels sous-jacents d'un groupe et ses relations de pouvoir. (Accompagnement Conseil RH)

- Intelligence émotionnelle : Capacité d'utiliser le pouvoir et la conscience des émotions (...), de sentir et d'utiliser efficacement l'information, l'appartenance et l'influence. (Michel Pretre)

- Influencer, intéresser, convaincre et persuader vos interlocuteurs par une écoute active, une flexibilité relationnelle qui intègre les motivations de l'autre. (LCA)

- Techniques d'identification des attentes de vos collaborateurs pour mieux ajuster votre message à leur sensibilité et créer un climat d'empathie et de performance. (LCA)

- Vos collaborateurs sont-ils en empathie (pour fidéliser vos clients) ? (Alpin)

- L'investissement sur l'amélioration des relations de proximité au sein de l'entreprise a des effets immédiats et de long terme sur le développement des relations et du dialogue avec les clients : l'intimité client. (Eurostaf)

\section{La relation à soi au service de l'efficacité professionnelle :}

- Nous devons nous entraîner à nous rencontrer nous-mêmes, à être en relation de manière efficace avec les autres sans renoncer à nous-mêmes ni mettre en danger nos relations de travail. (Monika Radecki) 
- Former les individus à connaître et à appliquer les techniques de communication ainsi que les normes de comportement et de jugement (...) pour une meilleure efficacité professionnelle ainsi que pour une réalisation de son potentiel personnel. (Solutions \& Co)

- Habiletés qui permettent à un individu (...) de se différencier, de se séparer de la masse et de réussir. (Solutions \& Co)

- Déterminer sa personnalité et celle des autres pour se faire une place. (Apollo)

\section{Le leadership}

- L'énergie positive que vous irradiez attirera les gens vers vous. (PMC)

- Utiliser la position d'hélicoptère pour identifier la cible, les enjeux, le contexte. (Cegos)

- Développer l'aisance face à la prise de parole pour aller plus loin dans l'expression de votre autorité et de votre charisme. (LCA)

- En décidant d'inscrire (vos collaborateurs) à ce séminaire, vous allez passer de convaincre à persuader. (...). Ils constateront par eux-mêmes l'amélioration constante de leur impact sur leur auditoire. (Alpin)

- Aider les managers à renforcer leurs compétences relationnelles pour accroître leur leadership. (Eurostaf)

Linfluence sur autrui constitue ainsi la référence transversale aux différents registres de CRS, et plus largement, la référence nécessaire à la consistance globale de la notion. Elle témoigne de l'ancrage constant de ces compétences dans un modèle entrepreneurial, avec pour trame implicite l'efficacité managériale. En définitive, le flou de la notion ne se situerait pas tant au niveau conceptuel que terminologique (décalage entre ce dont on parle et les labels qu'on lui appose). De ce point de vue, la référence au relationnel aurait une fonction de légitimation, nécessaire à la construction de la valeur sociale d'un modèle éthiquement discutable.

\section{Confrontation à la définition des CRS en contexte scientifique}

Dans les articles scientifiques, la distance à la personnologie est généralement plus marquée que dans les offres. Différentes dimensions descriptives y sont clairement distinguées. Une revue synthétique des définitions scientifiquement admises des compétences sociales est par exemple proposée dans Dépret \& Filisetti (2001), dans la perspective d'une remédiation aux nombreux biais, socio-affectifs et socio-cognitifs, présidant à l'évaluation d'autrui. Trois dimensions (facteurs) y sont dégagées :

- le « degré de compréhension de l'autre, du sens de l'interaction et de l'événement social»; 
- le « degré d'adhésion ou de respect des règles, normes et rôles sociaux en vigueur dans un groupe social donné »- et les auteurs insistent ici, par delà les références de la littérature, sur "l'importance des normes sociales relatives aux comportements d'interaction sociale » (p. 311) ;

- le « degré de retenue et de contrôle de soi »- cette troisième dimension étant plus marginalement mentionnée dans la littérature que les précédentes (p. 309).

Par delà ces dimensions, les auteurs distinguent une acception générale de la notion : «savoir faire social et capacité relationnelle », et une acception plus spécifique, comme " capacité à faire bonne impression, à susciter approbation sociale et affection» (p. 311).Nous ne relèverons pas les écarts systématiques entre approche scientifique d'une part, définitions d'usage dans les pratiques d'autre part, d'autant que sur les référents explicites eux-mêmes, ces écarts paraissent relatifs. Mais dans le corpus des offres, le modèle entrepreneurial amène une distorsion générale dans la caractérisation des CRS, tout comportement relationnel tendant à être interprété en rapport avec une visée d'influence unilatérale sur autrui. Cela étant cette interprétation pourrait peut-être trouver une assise scientifique dans un domaine directement concerné par le monde de l'entreprise. Pour examiner cette question, nous proposons l'analyse d'un article de référence en gestion, celui de Persais (2004). Cet article conjugue perspectives théoriques et appliquées dont la validité scientifique paraît établie, et il fait l'objet de nombreuses citations dans la discipline. L'auteur y propose une définition des CRS en tant que compétences stratégiques. L'influence sera donc explicitement au cœur de la perspective. Les CRS sont vues sous l'angle de l'alliance entre entreprises, et dites «stratégiques » en ce qu'elles contribuent à la performance, par le biais notamment d'une " meilleure intégration au sein de l'environnement».

Processus d'influence et visée utilitariste sont traités ici de front. En même temps, l'auteur défend une éthique de l'influence - sans pour autant aborder directement la question. Ainsi, bien qu'insistant sur la coopération entre partenaires pour une nouvelle organisation des échanges, ou encore sur l'importance des réseaux relationnels et la détection des "acteurs-clefs ", il se prémunit soigneusement contre toute évocation de pratiques douteuses en la matière (accord illicite entre firmes, lobbying...). Face à ces omissions, les divers partenariats explicitement cités se situent tous sur le registre du socialement acceptable, et même, pour nombre d'entre eux, du socialement valorisé (organismes et associations d'intérêt public, organisations écologistes tout particulièrement, mais aussi sécurité alimentaire, prévention des risques, associations de défense des consommateurs, défense des salariés, etc.). Préoccupations éthiques et stratégies persuasives à visée de profit ne seraient pas seulement conciliables : elles iraient de pair. Le document 3 reproduit deux extraits illustrant les stratégies discursives visant à l'assimilation entre ces deux ordres de préoccupations. Y sont soulignées toutes les références directement 
stratégiques, reflétant l'issue pré-déterminée de toute forme de négociation, dans un entourage sémantique mettant l'accent sur la responsabilité éthique de l'entreprise.

\section{Document 3}

\section{Stratégies persuasives et souci éthique : le brouillage des pistes.}

\section{Exemple 1 (p. 134)}

(...) la présence d'un « observatoire social » traduit une attention particulière portée aux salariés et aux organisations chargées de promouvoir les avancées dans le domaine social. La nomination d'un chargé de mission « environnement » signifie, tout au moins en partie, une volonté de favoriser le dialogue entre l'entreprise et les principales parties prenantes concernées (populations environnantes, organisations écologistes, pouvoirs publics). Plus généralement, dans le contexte actuel, l'entreprise doit s'efforcer de multiplier les contacts avec l'extérieur de manière à déterminer, en toute connaissance de cause, les groupes à « privilégier » dans le cadre d'une politique partenariale.

\section{Exemple 2 (p. 137)}

(...) Une attitude volontariste conduit l'entreprise à agir, de manière à faire perdurer ce lien, y compris en dehors de toute sollicitation de la part des acteurs concernés. Une firme du secteur chimique pourra, par exemple, être à l'initiative de rencontres avec les groupes écologistes et les populations environnantes. Elle les informera régulièrement, voire devancera leurs attentes en matière de sécurité et de prévention des risques. Elle s'efforcera de répondre à leurs exigences, quand bien-même celles-ci dépasseraient le cadre des contraintes légales ou réglementaires. L'entreprise doit donc reconnaitre la légitimité d'intérêts externes et adopter une démarche véritablement constructive vis-à-vis des groupes externes.

Plus fondamentalement, c'est la relation d'influence elle-même qui, dans son principe, est posée comme coopération moralement irréprochable - moralité conditionnant sa rentabilité : « orientation positive », « confiance réciproque », plutôt que " défiance » et " ambitions sournoises » sont " évidemment " plus rentables pour chaque acteur. Relevons encore les références à la « loyauté » p. $131, l^{\prime}$ ' intégrité » p. 131 et p. $137 .$. Ce discours moral permet au passage de rappeler l'intérêt commun des partenaires : éviter « la mise en place de mécanismes de contrôle »- rien n'étant dit de l'intérêt de cet évitement, évidence au cœur de la doxa du libéralisme économique.

Parailleurs, siles compétences stratégiques sont définies comme compétences de l'entreprise, elles n'en sont pas moins décrites comme "individuelles ». Paradoxe ? L'auteur souligne que l'idée de " compétence collective », dans la 
littérature, est peu présente (p. 123) ; le terme même pourrait constituer un «abus de langage ». Et quand la notion est utilisée (en particulier dans l'analyse de stratégies), elle est définie "par analogie aux compétences individuelles ». La personnologie revient ici à la charge : référence aux "volontés », aux « talents », désignation du savoir-être comme "volonté »... Les CRS sont néanmoins conçues comme ancrées dans l'organisation (pp. 123 sq.), mais le dépassement d'un niveau d'analyse intra-individuelle semble difficilement concevable dans cette littérature, et si la notion de " compétence collective " n'est pas totalement exclue, elle se réfere toujours, dans sa description opérationnelle, aux réseaux relationnels - et non à des relations intersubjectives. La conception psychologisante des CRS se traduit également, et là peut-être plus qu'ailleurs, par l'intérêt que l'auteur fait porter sur la personnalité du manager : caractérisé par un idéal «naturel» (p. 137) de figure de pouvoir, son intégration du « code informel » de l'entreprise à sa propre personnalité est censée aller de soi dans la plupart des cas, une " politique de formation adaptée » pouvant remédier aux éventuels cas de « léger déphasage » (p. 138). Les CRS apparaissent donc bel et bien comme une dimension de la personnalité, voire : la personnalité elle-même, et leur description comme indissociable de la valeur accordée au modèle entrepreneurial. Ce qui n'est pas sans générer quelques contradictions, et ce faisant, assigner des objectifs paradoxaux aux pratiques d'évaluation et de formation.

\section{La gestion des paradoxes dans la formation aux CRS : entre perversion et aliénation?}

La notion de CRS renferme une injonction paradoxale : être tout à la fois allégeant et autonome. L'adaptation à l'entreprise, condition de l'autonomie, exige en fait une identification à ses valeurs, normes, objectifs, etc. qui serait vécue comme soumission (source de risques psycho-sociaux, notion permettant de faire l'impasse sur la question de l'aliénation) si la personne ne parvenait pas à se convaincre de sa propre autonomie. Les formations aux CRS constituent en quelque sorte des stages de "remise à niveau " amenant les sujets à " incorporer (...) les mots, les formulations et les pensées légitimes, parce que légitimés et légitimants dans le milieu social et/ou professionnel ", ce que l'on peut traduire en termes de «maltraitance symbolique» (Diet 2009 : 80). Le corrélat de l'illusion de l'autonomie de l'agent exécutant pourrait bien être le développement de conduites perverses en matière de management, en lieu et place de conduites autoritaires devenues contre-normatives. Pour Diet par exemple, « le stress, le chantage et la menace narcissique sont devenus des instruments banalisés du management », et « leur maîtrise et leur emploi font aujourd'hui partie des "qualités" exigées des cadres » (2010:65).

Faut-il alors rendre compte des enjeux propres à la notion de CRS en termes d'aliénation ? Celle-ci peut être définie comme l'ensemble des processus contribuant à la construction d'une identité artéfactuelle (identification 
à un modèle normatif à forte valeur sociale), et consistant pour l'essentiel à attribuer à ses propres conduites des significations conformes à ce que le fonctionnement social exige pour asseoir sa légitimité. Et la notion de CRS touche à l'identité même de la personne. Bézille et Astolfi le relevaient déjà en 1999 à propos de la notion de la compétence en général : « Reconnaître à une personne telle ou telle compétence revient à lui attribuer une identité, plus ou moins valorisée » (p. 16). Or cette attribution identitaire se trouve d'autant plus marquée que les compétences en question relèvent des CRS.

À un niveau social et sociétal, ce processus contribuerait à accroître le fossé entre normes qui régissent les comportements, et normes qui régissent les discours. Or, la focalisation sur le relationnel semble relever de ce second registre ; non qu'elle n'entretienne aucun rapport motivé avec les comportements relationnels effectifs, mais elle permet de les labelliser de telle sorte que leur signification consciente pour les sujets ne corresponde pas au vécu d'une relation qui n'a objectivement pas grand chose à voir avec ce qu'elle prétend être. En somme, si la notion de compétence en général favorise l'occultation des facteurs sociaux au profit d'une focalisation sur les caractéristiques psychologiques, ce serait plus spécifiquement la relation à l'autre que la notion de CRS psychologise, c'est-à-dire désocialise, en la plaçant sous l'entière détermination de qualités individuelles visant l'instrumentalisation. Et de fait, les références à la communication dialogique sont marginales dans les discours sur les CRS, au profit d'une focalisation sur la capacité de maîtrise de relations artificiellement bilatérales.

Compte-tenu de l'exigence de production d'une impression favorable, avec mise en avant de la maîtrise de soi, l'implication de la sphère affective, aussi bien dans le rapport à l'autre qu'à soi, est là encore source potentielle d'aliénation, en tant que seule gestion possible d'un paradoxe : s'impliquer affectivement dans la relation, être authentique (valorisation de l'expression de soi), et se sentir bien... à des fins stratégiques. Le discours normatif sur "l'intelligence émotionnelle », qui dans les usages managériaux se substitue souvent à la notion de CRS, s'appuie certes sur un domaine scientifique très investi actuellement et dont les résultats sont de fait probants. Mais l'accent est surtout mis, dans le domaine managérial, sur l'expression des affects - plutôt que sur les émotions elles-mêmes. En d'autres termes : les affects doivent faire l'objet d'une expression socialement acceptable, expression à visée stratégique : capacité à signifier son écoute et son approbation, par exemple, n'implique pas écouter et approuver. Rien à voir évidemment avec une éthique humaniste de la bienveillance; pas plus au demeurant qu'avec l'intelligence émotionnelle, à strictement parler. Car il ne s'agit pas tant d'utiliser ses émotions pour mieux comprendre la relation, que de comprendre la relation pour déterminer ce qu'il convient de montrer que l'on éprouve, et faire inférer à l'autre la positivité de son propre état affectif, positivité posée implicitement comme condition d'une influence efficace. En pareille position, quelle alternative à l'aliénation, 
si ce n'est la perversion ? Auquel cas, les CRS serviraient au premier chef la normalisation de conduites manipulatrices, sous couvert d'un psychologisme humaniste fonctionnant comme caution déontologique.

Les formations aux CRS sont désormais incluses dans de multiples programmes de formation centrés sur la manière d'être des personnes. Tous ont en commun de se cibler sur l'individu, et visent à lui apprendre à se présenter et se comporter socialement de façon plus conforme au regard des exigences de son environnement social, professionnel en particulier. En même temps, le « développement personnel » est censé générer un mieux-être psychologique. Il s'agit en somme de supprimer le hiatus entre ce que l'on est, et ce que l'on doit être. De manière générale, c'est la relation à l'environnement social qui est conçue comme reposant sur un déterminisme personnologique fort : savoir influencer l'autre, agir sur l'autre. L'adaptation, comme le changement, ne peuvent relever ici que de processus unilatéraux... L'action sur l'environnement est donc toute entière sous la dépendance d'un potentiel individuel d'action persuasive sur autrui, d'où l'enjeu fondamentalement politique porté par la notion de CRS.

On pourra objecter que les $\mathrm{CRS}$ sont parfois décrites précisément en termes de compétences politiques, ou du moins, que ces dernières en font partie, par exemple chez Ferris, Fedor, \& King (1996). Les compétences politiques, indispensables au manager et composant son "style interpersonnel », relèveraient d'un savoir communiquer dans lequel l'image de soi est centrale : se montrer agréable, inspirer confiance, donner une impression de sincérité... ce dans le but de construire un réseau relationnel important. Or, l'usage du terme «politique » ici, tout comme la référence à la communication, s'inscrivent dans une sorte de CRS générique conforme à ce que nous avons rencontré jusqu'à présent. Négociation, dialogisme, communication intersubjective, construction d'un espace symbolique commun... sont exclus de cet univers de référence.

En somme la référence au relationnel, dans le contexte des CRS, constitue un filtre idéologique légitimant l'individualisme utilitariste, en le présentant comme antithétique de la soumission à un environnement professionnel devenu non négociable. La communication telle qu'elle transparaît ici traduit l'impuissance de l'inter-action avec l'autre, c'est-à-dire de la communication à strictement parler, et ce faisant de l'action collective. Faut-il alors s'étonner, à l'instar de Dubois \& Charpentier, de « constater en entreprise la déconnexion manifeste et récurrente entre communication et régulation sociale, comme si la communication n'avait pas à intervenir dans ce domaine, comme si elle devait rester en permanence dans un registre infrasocial ou métasocial » $(2006: 15)$ ? 


\section{Les CRS, substitut de la communication}

Limportance accordée aux CRS témoigne de la naturalisation psychologisante caractérisant l'individualisme, en même temps que de la normativité de la communication. Or, comme le remarque Sfez, "on ne parle jamais autant de communication que dans une société qui ne sait plus communiquer avec elle-même.(...) On ne parlait pas de communication dans l'Athènes démocratique, car la communication était au principe même de la société » (1988: 28). Ce paradoxe ne révèle-t-il pas in fine le caractère pathologique des relations sociales auxquelles réfère la notion de CRS ? Le concept de tautisme forgé par Sfez est à cet égard éclairant (document 4) (voir Camus 2007 : 293 sq.)

\section{Document 4}

\section{Le tautisme}

Le concept complexe de " tautisme ", contraction de " tautologie " et " autisme », que propose Sfez dans sa Critique de la communication, rend compte de l'idéologie de la communication en tant que "forme symbolique », qui caractériserait nos sociétés. Le tautisme se définit par :

- la confusion des registres de la communication (réalité représentée et réalité exprimée), confusion que Sfez qualifie de "primordiale et source de tout délire » $(1988: 17)$;

- la vacuité de l'autoreprésentation de la société (tautologie : formule autoréférente donc vide de sens ; en se désignant comme "société de communication ", la société ne dit rien de plus que : "Je suis société " (p. 101);

- l'isolement quasi autistique que produit le système de communication;

- " tautisme " évoque également une "visée totalisante, voire totalitaire ", que Sfez évoque ainsi : « la glu qui me colle à l'écran, la réalité de la culture écranique, réalité toujours médiée alors qu'elle s'exhibe comme réalité première » (p. 17).

Ainsi, le registre expressif est au cœur des CRS, l'image de soi en constituant le noyau dur. Et en définitive, ce à quoi réfèrent globalement les compétences sociales, c'est à un potentiel de valorisation, qui aura pris le pas sur la valeur substantielle du travail. Or, c'est pourtant le travail lui-même qui constitue l'objet premier de la communication en contexte professionnel, sa matière, la " réalité représentée » sur laquelle les partenaires de l'échange se doivent de s'accorder. Et la notion de CRS n'est qu'accidentellement rapportée à la communication opératoire (voir Camus 2012), centrée sur l'activité en situation ; elle ne semble pas concerner ces échanges apparemment anodins, et dans lesquels la valeur de la coopération est indissociable de l'exécution de 
la tâche, la réciprocité de l'influence étant la condition de l'ajustement des représentations mutuelles.

En somme, la communication version CRS est une communication sans objet, dans laquelle le rapport au réel devient problématique (source de " délire ", pour reprendre le terme de Sfez).

Par ailleurs, la notion de CRS, et plus largement le culte de l'autonomie individuelle et de l'indépendance de l'individu à l'égard du social, entretient une représentation dichotomique entre ce qui relèverait du psychologique et ce qui relèverait du social. Or, cette dichotomie n'est pas sans rapport avec la vacuité de l'autoreprésentation de la société que souligne par exemple Castoriadis : «Il ne peut pas y avoir de société qui ne soit pas quelque chose pour elle-même ; qui ne se représente pas comme étant quelque chose (...) Tout individu doit être porteur (...) de cette représentation de soi de la société. C'est là une condition vitale de l'existence psychique de l'individu singulier. Mais (...) il s'agit aussi d'une condition vitale de l'existence de la société elle-même » (1996: 20 sq.).

Paradoxe ultime : les CRS préconisent l'indépendance à l'égard d'autrui. En matière de relations sociales, l'influence sur autrui, la résistance aux pressions, la maîtrise de soi, l'utilisation stratégique des émotions... ne traduisent-elles pas une injonction implicite à ne pas être affecté par l'autre ? Ce qui n'est pas sans rappeler le constat d'Enriquez \& Haroche : les démocraties libérales favoriseraient le développement de personnalités apathiques, c'est-à-dire d'individus en état d' «impossibilité d'être troublés » (2002:74), développement symptomatique du processus pathologique dans lequel nos sociétés seraient engagées.

Enfin, si l'on s'accorde sur la fonction primordiale de la communication : convaincre l'autre pour co-construire du sens, c'est à l'interprétation sans laquelle «il ne saurait être de parole échangée ", que la confusion tautistique porte atteinte (Sfez, op. cit. : 470). La conception des relations sociales qui sous-tend la notion de CRS apparaît sous cet angle comme l'antithèse de la communication. Elle contribuerait bien plutôt à la " désymbolisation ", cette « forme inédite de domination » que décrit Dufour (2003, en particulier 225 sq.). Or, parce que la fonction symbolique est ce par quoi le sujet peut se construire en tant que sujet, la désymbolisation signe l'avènement du " sujet post-moderne », " précaire, a-critique et psychotisant » (p. 24 sq.) La formation aux CRS, en dénaturant la communication, risque bien d'apporter sa contribution à cette entreprise.

\section{BIBLIOGRAPHIE}

ALTHUSSER L., "Idéologie et appareils idéologiques d'état ", La Pensée, 1970, $n^{\circ} 151,3-38$.

BEAUVOIS J.-L., La psychologie quotidienne, Paris, PUF, 1984, 211 p. 
BEAUVOIS J.-L., Traité de la servitude libérale. Analyse de la soumission, Paris, Dunod, 1994, 247 p.

BEAUVOIS J.-L., Les illusions libérales, individualisme et pouvoir social. Petit traité des grandes illusions, Grenoble, PUG, 2005, 424 p.

BÉZILLE H., ASTOLFI J.-P., «Identités et compétences dans les métiers de l'éducation et de la formation ", Questions de Recherches en Éducation, 1999/1, p. 9-21.

CAMUS O., "Idéologie et communication », dans PÉTARD J.-P. (dir.), Psychologie sociale, Paris : Bréal, 2007, p. 269-334.

CAMUS O., «L'évaluation des compétences relationnelles et sociales : obstacles idéologiques et reconceptualisations nécessaires ", Education Permanente, 2012 (à paraître)

CASTORIADIS C., La montée de l'insignifiance (les carrefours du labyrinthe IV), Paris, Seuil, 1996, 244 p.

DAMASIO A.R., L'erreur de Descartes. La raison des émotions, Paris, Odile Jacob, 1994 (tr. fr. 1995), 368 p.

DÉPRET E., FILISETTI L., « Juger et estimer la valeur d'autrui : des biais de jugement aux compétences sociales ", L'orientation scolaire et professionnelle, 2001 Vol. 30, $\mathrm{n}^{\circ} 3$, p. 297-315.

DIET E., " Management discours de l'emprise, idéologie et désubjectivation ", Connexions, 2009-1, n 91, p. 65-83.

DIET E., «Le désert des barbares. Notes sur la destructivité de l'hypermodernité néolibérale », Connexions, 2010-2, n 94, p. 61-83.

DUBOIS C., CHARPENTIER J.-M., "Communication, interaction et action ", Éducation permanente, 2006-2, n 167, p. 11-22.

DUFOUR D.-R., L'Art de réduire les têtes. Sur la nouvelle servitude de l'homme libéré à l'ère du capitalisme total, Paris, Denoël, 2003, 254 p.

ENRIQUEZ E., HAROCHE C., La face obscure des démocraties modernes, Ramonville-Saint-Agne, éditions érès, 2001, 119 p.

FERRIS G.R., FEDOR D., KING T.R., «A political conceptualization of managerial behavior ", Human Resource Management Review, 1996, n4-1, p. 1-34.

FOUCAULT M., L'Archéologie du Savoir, Paris, Gallimard, 1969, 279 p.

PERSAIS E., "Les compétences relationnelles peuvent-elles s'avérer stratégiques ? », Revue Française de Gestion, 2004/1, n 158, p. 119-145.

SFEZ L., Critique de la communication, Paris, Seuil, 1988 (éd. 1992), 527 p.

\section{Annexe : corpus des offres (nom et type de prestation)}

- Accompagnement Conseil RH.

- Alain Martin. Conseil, formation et coaching en management.

- Alpin. Activateur de Performance Relationnelle ${ }^{\circledR}$ (formation exclusive aux compétences relationnelles).

- Apollo formation. Formations professionnelles pour l'entreprise.

- Cegos. Formation professionnelle et continue. 
- Docteur Yves Doutrelugne (formation exclusive à la compétence relationnelle).

- Eurostaf (groupe Les Échos) (études de marché, études marketing, études stratégiques, diagnostics de groupes).

- Flück-Compétences. Ressources humaines et compétences.

- GKCF - Consulting - Formation - Coaching.

- Horizon Poitou-Charentes - GIP AGEVIF formation*.

- Institut du self-coaching - Coaching - Formations managériales.

- Ithaque Coaching. Coaching des odyssées professionnelles*.

- LCA Performances LTD - Formation RH.

- Marc Thomas consultant. Conseil / Accompagnement / Formation professionnelle.

- Michel Pretre - Management \& pre-sales.

- Monika Radecki - Conseil en communication.

- ORSYS formation - La formation à l'informatique et au management.

- PMC - SA - révélateur de potentiels (formations, coaching)

- Sedifop. Service Diocésain de Formation Permanente.

- Solutions \& Co. Formations en compétences relationnelles organisationnelles.

- TQ16 - Formation.

- *offres dont la conception des CRS est apparue atypique au regard du reste du corpus, et dont les résultats présentés ici ne rendent pas compte (soit $9,5 \%$ du corpus).

Résumé : Le modèle des compétences, maintes fois décrit comme produit idéologique de l'individualisme libéral, tend à se réorganiser autour de la notion de compétences relationnelles. Par delà la confusion apparente qu'apporte cette notion polysémique, une analyse de corpus conçu en tant que formation discursive révèle sa consistance paradoxale : autonomie supposant l'identification à l'entreprise, empathie instrumentalisante, relégation du collectif dans l'interpersonnel... Ces compétences apparaissent in fine comme source d'aliénation, et plus largement substitut tautistique de la communication. Mots-clés : Compétences relationnelles, compétences sociales, communication, idéologie, aliénation, tautisme.

Abstract : The notion of social skills: a psychotic view of the interpersonal relationship? The competence model, commonly described as an ideological product of liberal individualism, is reorganizing itself around the notion of interpersonal skills. Beyond the seeming confusion of this polysemic concept, a corpus - analysed as a discursive formation - reveals its paradoxical consistency: autonomy assuming identification to the corporation, instrumentalised empathy, collective matters being reduced to interpersonal matters... These skills eventually appear as a source of alienation, and more generally as a tautistic surrogate of communication.

Keywords : Interpersonal skills, social skills, communication, ideology, alienation, tautism. 